\title{
Dare voce all'incompetenza: un grande male del nostro tempo
}

\section{Claudio Marazzini}

PUBBLICATO: 09 GENNAIO 2020

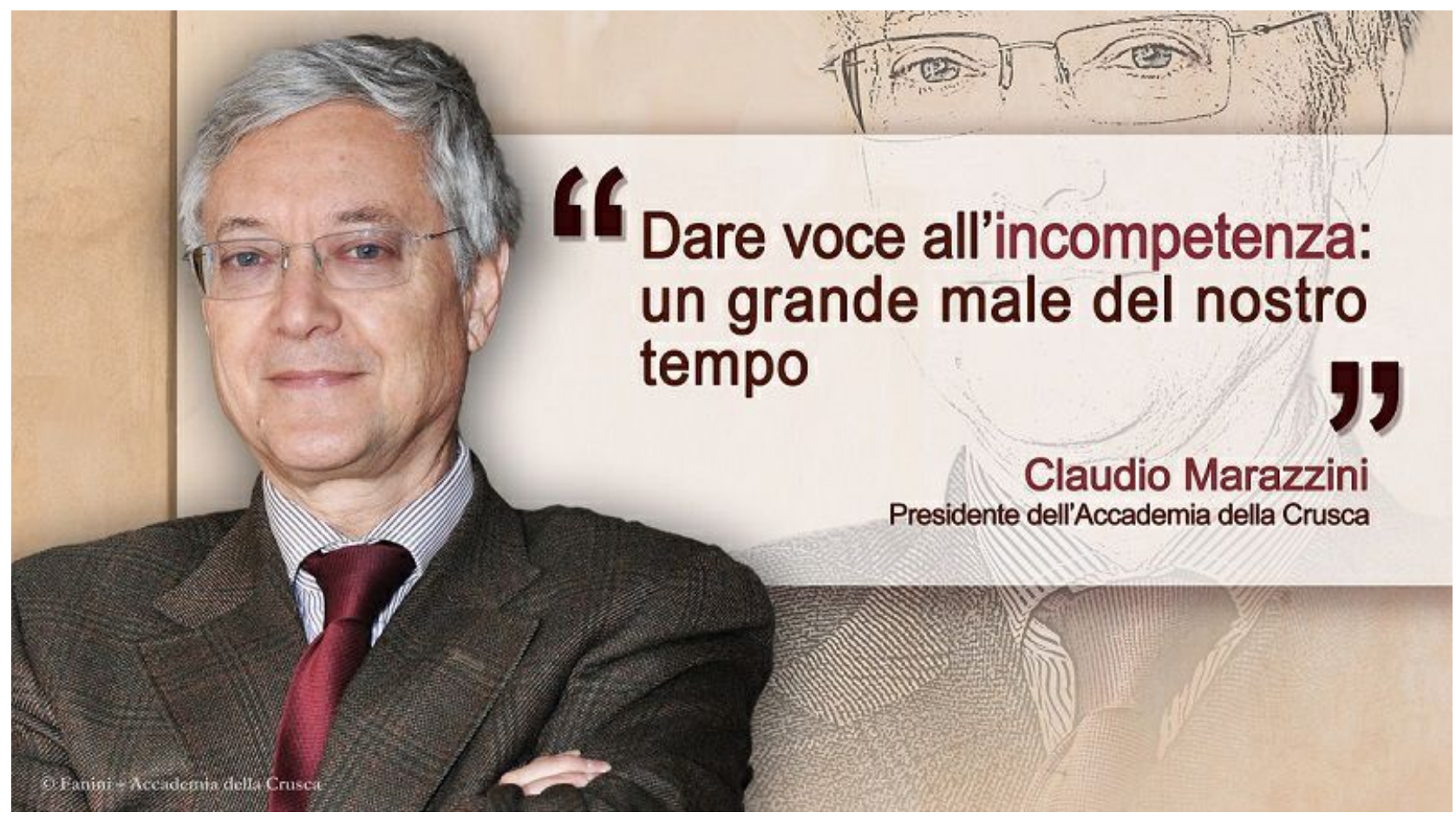

$\mathbf{L}^{\prime}$

Accademia della Crusca in questi giorni ha cambiato il fornitore del servizio di rassegna stampa. A beneficio di chi non s'intende di queste cose, preciserò che i servizi di rassegna stampa forniscono ogni giorno all'abbonato la raccolta completa dei ritagli giornalistici e web che lo menzionano. L'Accademia ha appunto attivato un servizio del genere, come del resto molti istituti, ditte, organizzazioni interessate alla ricaduta mediatica della propria attività. Il presidente dell'Accademia della Crusca, naturalmente, non legge ogni giorno la rassegna stampa completa, della quale si occupa l'addetto dell'Ufficio stampa. Tuttavia, visto che era stato attivato un nuovo abbonamento, oggi ho dato un'occhiata, anche per verificare l'efficienza del sistema. L'occhio mi è caduto sull'articolino che riproduco qui in allegato, di cui non dirò la fonte, per non fare pubblicità a chi non la merita. Sono andato immediatamente a verificare se la testata giornalistica che aveva accolto questa letterina l'avesse in qualche modo commentata, per esempio spiegando che la "i" in questione è parte della desinenza del verbo. Fra l'altro, l'intervento della Crusca sull'argomento (si veda, sul sito dell'Accademia, Desinenze -iamo, -a mo nella prima persona plurale dell'indicativo presente) non esprime una posizione puristica a favore della forma con la " $i$ ", che molti considerano più corretta, ma le ammette entrambe! Quindi la requisitoria - suprema leggerezza - non si è accompagnata nemmeno alla verifica dell'opinione reale espressa dalla Crusca sulla materia. Si aggiunga che sarebbe stato possibile rinviare l'incompetente lettore al sito web della Treccani, se non si voleva la Crusca. Sarebbe bastato consultare un qualunque dizionario. Invece è stata pubblicata con molta visibilità un'opinione priva di fondamento. Il lettore, invece di esibire quella grande aria di scandalo, con il tono del Solone moralizzatore, avrebbe potuto trovare facilmente la risposta consultando una qualunque grammatica scolastica. Sarebbe stata per lui la buona occasione per stare zitto e imparare qualche cosa. Invece ha assunto il tono del gran sapiente offeso, ha fatto ricorso alla propria fallace memoria, ha tirato in ballo gli insegnamenti di un'antica maestra, probabilmente 
innocente, ma certo impossibilitata a smentire. Roba da farci una risata, insomma. Tuttavia l'intervento mi ha colpito, e per questo l'ho voluto denunciare pubblicamente: è un caso esemplare della condizione di oggi, in cui radio, televisioni e giornali con estrema facilità danno voce e spazio all'ignoranza e all'incompetenza, anche in casi in cui non sarebbe difficile verificare la verità. Così, a scopo diaudience, si favoriscono spostati d'ogni genere, terrapiattisti, agitatori, sostenitori di balordaggini che, con prosopopea incredibile, con stupefacente sicurezza, privi di ogni autocritica e di ogni controllo, lanciando accuse senza pudore, nella certezza dell'impunità, in una società permissiva, anzi dedita al culto dell'imbecille. A mio giudizio, questo costituisce un reale pericolo sociale, da cui la stampa dovrebbe difenderci, anziché favorire il fenomeno.

Alla domanda "Ma la Crusca è infallibile?", posta come attraente titolo allintervento del saccente lettore, possiamo dunque rispondere che certamente la Crusca può sbagliare, come chiunque altro al mondo; però, in questo caso, non ha sbagliato, e la cosa era di assoluta evidenza. E allora, ecco la responsabilità del giornale: perché concedere tanto spazio a chi metteva ingiustamente e pretestuosamente sotto accusa un'istituzione prestigiosa con un argomento risibile? Forse era proprio questo il punto di interesse: parlar male di qualcuno importante, lasciando intuire al lettore che la Crusca insegna cose sbagliate. Una persona di senno avrebbe almeno svolto qualche controllo preventivo per verificare come stessero davvero le cose. Invece, nulla. E poi ci dicono che i giornali ci salvano dal diluvio delle fake news, le bufale incontrollate della Rete...

Scorrendo la medesima rassegna stampa, mi è caduto l'occhio su di un altro articolino, in cui si metteva nuovamente sotto attacco la Crusca: un pezzo uscito sul "Resto del Carlino" il 3I dicembre, l'ultimo dell'anno 2019. Sotto il titolo "Non sappiamo cosa accadrà" (forma meno elegante, al posto del più corretto "che cosa accadrà") si censurano i tentativi di pronosticare gli avvenimenti dell'anno successivo. L'autore dell'articolo, tuttavia, non si limita a svolgere questa buona missione contro gli indovini, ma se la prende con la Crusca che non c'entra per nulla. Scrive costui: "Come l'Accademia della Crusca con le forme linguistiche scorrette (esempio: "esci il cane"), cosi anche certi giornali talvolta favoriscono la stupida curiosità dei lettori". C'è da chiedersi chi sia lo stupido, per la verità. Ostinatamente, nonostante tutte le smentite, si torna a far credere che la Crusca abbia accolto (e dove, poi?) la forma transitiva "Esci il cane". Non si è dunque capito niente delle discussioni che si sono accese mesi fa, né ci si è curati di quello che l'Accademia ha scritto, precisato e ripetuto. La faciloneria e la grossolanità vincono, ancora una volta. Ne prendiamo atto.

Purtroppo alcuni giornali (si spera non tutti) sentenziano sulle cose che non capiscono. Ce ne accorgiamo, noi della Crusca, per i problemi di lingua; ma, a questo punto, ci assale il timore che lo stesso fenomeno si ripeta per molti altri argomenti, della politica e della vita civile. Il danno non sarebbe da poco.

\section{Cita come:}

Claudio Marazzini, Dare voce all'incompetenza: un grande male del nostro tempo, "Italiano digitale", 2020, XII, 2020/1 (gennaio-marzo)

DOI: $10.35948 / 2532-9006 / 2020.3301$

Copyright 2020 Accademia della Crusca Pubblicato con licenza creative commons CC BY-NC-ND 